\title{
Evaluation of Antioxidant Ability In Vitro and Bioavailability of trans-Cinnamic Acid Nanoparticle by Liquid Antisolvent Precipitate
}

\author{
Wengang Li, Xiuhua Zhao, Xiaoli Sun, Yuangang Zu, Ying Liu, and Yunlong Ge \\ Key Laboratory of Forest Plant Ecology Ministry of Education, Northeast Forestry University, Harbin, Heilongjiang 150040, China \\ Correspondence should be addressed to Yuangang Zu; yuangangzu@163.com
}

Received 9 January 2016; Accepted 29 February 2016

Academic Editor: Michele Laus

Copyright (C) 2016 Wengang Li et al. This is an open access article distributed under the Creative Commons Attribution License, which permits unrestricted use, distribution, and reproduction in any medium, provided the original work is properly cited.

\begin{abstract}
TCD is a kind of organic acid that is isolated from cinnamon bark or benzoin. TCD has significant antioxidant activity and is widely used in pharmaceutical, cosmetic, and food additives. But TCD has shortcomings of low bioavailability due to poor water solubility. Therefore, we use ethanol as a solvent, deionized water as antisolvent, and hydroxypropyl methylcellulose (HPMC) as the surfactant to prepare TCD nanoparticle powder. The optimum preparation conditions were determined as follows: TCD-ethanol solution concentration was $170 \mathrm{mg} / \mathrm{mL}$, the volume ratio of antisolvent was 4 times that of solvent, and the amount of the surfactant was $0.3 \%$ stirred for $10 \mathrm{~min}$ by $2500 \mathrm{rpm}$; TCD nanoparticle with a mean particle size (MPS) of $130 \pm 12.5 \mathrm{~nm}$ is obtained under the optimum conditions. SEM, FT-IR, LC-MS/MS, XRD, and DSC were used to characterize the TCD nanoparticle. The results showed that the chemical structure of TCD nanoparticle was not changed, but the crystallization was significantly reduced. Solubility, dissolution rate, antioxidant activity, the in vitro transdermal penetration, and bioavailability of TCD nanoparticles were all much better than these of the raw TCD. These results suggested that TCD nanoparticle might have potential value to become a new oral or transdermal TCD formulation with high bioavailability.
\end{abstract}

\section{Introduction}

trans-Cinnamic acid (TCD, also known as trans-3-phenylacrylic acid) is one of the main chemical constituents of cinnamon which belongs to plant Lauraceae $[1,2]$. This acid possesses antibacterial, elevated white blood cell, cholagogue, and anticancer effects [3]. It can also inhibit melanoma and proliferation and promote differentiation of cancer cell in vitro $[4,5]$. It may be indirectly combined with DNA to regulate the expression of certain genes and block the growth and proliferation of tumor cells without embryotoxicity performance for clinical treatment of tuberculosis [6]. TCD as a natural oral antioxidant has a significant antioxidant effect and plays an important role in clearing oxygen-free radicals of the body, delaying human aging, and improving immunity [7]. However, the application of TCD is restricted by its poor water solubility and low oral bioavailability.

Preparation of nanoparticles with small and uniform particle size distribution is an effective way to improve the bioavailability of poorly soluble drugs [7]. Preparation technology of nanoparticles mainly includes ball milling method, jet milling method, supercritical fluid technology, and liquid antisolvent precipitate. Mechanical pulverization methods, such as ball milling and jet milling, are widely used to prepare nanoparticle drugs, but their disadvantages include energy consumption, low efficiency, and a wide particle size distribution [8]. Supercritical fluid technology has several disadvantages, such as large investment in equipment, low yield, and difficulty for industrialization [9]. Liquid antisolvent precipitate is an important way to prepare drug nanoparticles [10]. It has potential prospects because of its low cost and easy operation compared with traditional preparation technology [11]. To date, numerous types of poorly soluble oral drugs have been successfully prepared by this method [12].

In the present work, we prepared TCD nanoparticle using liquid antisolvent precipitate, which has not been reported in literature. This work aimed to prepare the TCD nanoparticle 
TABLE 1: Single factor and levels program.

\begin{tabular}{lccccc}
\hline Levels & $\begin{array}{c}(\mathrm{A}) \\
\text { Precipitation } \\
\text { time (min) }\end{array}$ & $\begin{array}{c}(\mathrm{B}) \mathrm{TCD} \\
\text { concentration } \\
(\mathrm{mg} / \mathrm{mL})\end{array}$ & $\begin{array}{c}\text { Factors } \\
\text { antisolvent and } \\
\text { solvent }\end{array}$ & $\begin{array}{c}\text { (D) HPMC additive } \\
\text { proportion }(\%)\end{array}$ & $\begin{array}{c}\text { (E) Stirring intensity } \\
(\mathrm{r} / \mathrm{min})\end{array}$ \\
\hline 1 & 3 & 30 & $1: 1$ & 0.1 & 500 \\
2 & 5 & 50 & $2: 1$ & 0.2 & 1000 \\
3 & 10 & 100 & $4: 1$ & 0.3 & 2000 \\
4 & 20 & 150 & $6: 1$ & 0.4 & 2500 \\
5 & 30 & 170 & $8: 1$ & 0.5 & 200 \\
\hline
\end{tabular}

to improve dissolution rate, oral antioxidant, and bioavailability. Scanning electron microscope (SEM), Fourier transform infrared (FT-IR), spectroscopy liquid chromatography coupled with tandem mass spectrometry (LC-MS/MS), Xray diffraction (XRD), and differential scanning calorimetry (DSC) were used to characterize TCD nanoparticle. Solubility, dissolution rate, purity, transdermal penetration, antioxidant activity, and bioavailability of TCD nanoparticle were also evaluated.

\section{Experimental Section}

\subsection{Experimental}

2.1.1. Materials. trans-Cinnamic acid (mass purity $\geq 99 \%$ ) with a molecular formula of $\mathrm{C}_{9} \mathrm{H}_{8} \mathrm{O}_{2}$ and molecular weight of 148.16 and heparin were obtained from American SigmaAldrich Co., Ltd. Deionized water was purified by a Milli$\mathrm{Q}$ water purification system from Millipore (Bedford, MA, United States). Ethanol, methanol (chromatographic grade), acetonitrile (chromatographic grade), diphenylamine, glacial acetic acid, hydrochloric acid, and $\mathrm{KBr}$ (spectrographic grade) were provided by Tianjin Chemical Reagent Co., Ltd. Diphenylpicrylhydrazyl (DPPH) radical, hydroxypropyl methylcellulose (HPMC) (viscosity $=15 \mathrm{mPa} \cdot \mathrm{s}$ of $2 \%$ solution), phosphate buffered saline (PBS), mannitol, and Tween80 were purchased from Tianjin Bodi Chemical Co., Ltd. Pepsin was purchased from Sinopharm Chemical Reagent Co., Ltd. Other reagents were all of analytical grade.

2.1.2. Preparation of TCD Nanoparticle. Ethanol and deionized water were selected as solvent and antisolvent, respectively, to prepare TCD nanoparticles during liquid antisolvent precipitate. The experimental processes are illustrated in Figure 1. At room temperature, the raw TCD-ethanol solution was injected into the antisolvent with $0.3 \%(\mathrm{w} / \mathrm{w})$ HPMC by an injection pump (JZB-1800D; Jianyuan Medical Instrument, China) at an injection rate of $1 \mathrm{~mL} / \mathrm{min}$ stirring at a certain speed intensity, which resulted in a rapid formation of milky suspension. A small amount of suspension was then collected and diluted. Subsequently, a laser particle size analyzer was used to detect the mean particle size (MPS). The remaining suspension was centrifuged for $10 \mathrm{~min}$ under the condition of $10000 \mathrm{rpm}$; then residual ethanol was removed using deionized water by three times of washing and the precipitation was collected. $30 \%$ mannitol was added to

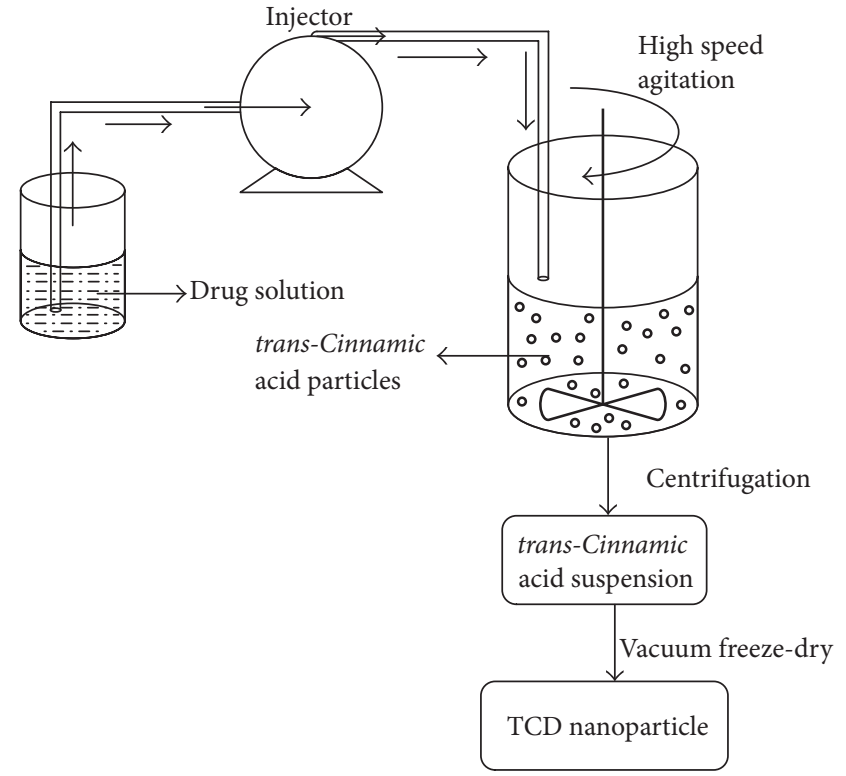

FIGURE 1: Diagram of the experimental processes to prepare the TCD nanoparticle.

the suspension as freeze-dried cryoprotector. Finally, TCD nanoparticles were obtained by prefreezing for $12 \mathrm{~h}$ at $-40^{\circ} \mathrm{C}$ in a refrigerator and then lyophilized at $-50^{\circ} \mathrm{C}$ for $48 \mathrm{~h}$. TCD nanoparticles were without mannitol under same freezedrying conditions.

2.1.3. Optimization of TCD Nanoparticle. Single-factor analysis was employed to optimize the TCD nanoparticle operating conditions by liquid antisolvent precipitate process. Through preliminary experiments, we selected the following five factors which had an arresting impact on the MPS of the TCD nanoparticle: precipitation time at 3-30 min, concentration of TCD-ethanol solution at $30-170 \mathrm{mg} / \mathrm{mL}$, volume ratio of antisolvent to solvent at $1: 1-8: 1$, amount of surfactant at $0.1 \%-0.5 \%$, and stirring intensity at $500-2500 \mathrm{rpm}$. The detailed level of each factor is presented in Table 1. A laser diffraction particle size analyzer was used to detect the MPS of the TCD nanoparticle and determine optimized conditions of the antisolvent precipitation process results. The optimum condition for every factor was selected based on the smallest MPS. 


\subsection{Characterization of TCD Nanoparticle}

2.2.1. Analysis of Morphology and Particle Diameter. The morphologies of the TCD powders were detected by SEM (Quanta 200; FEI, Netherlands). The samples were prepared by direct deposition of the powder onto a carbon tape placed on the surface of an aluminum tray and were coated with gold for 4 min using a sputter coater before analysis. The MPS of TCD nanosuspension was detected by DLS (ZetaPALS; Brookhaven Instruments, USA). TCD nanosuspension sample $(3 \mathrm{~mL})$ was added to the sample cell to detect the MPS. The sample was diluted with a mixture with the same composition as that during the antisolvent process if necessary. Each measurement was repeated at least three times.

2.2.2. FT-IR. FT-IR spectrum of the samples was obtained by IRAffinity-1 (Shimadzu, Japan) and recorded in the wavenumber range of $4000 \mathrm{~cm}^{-1}$ to $500 \mathrm{~cm}^{-1}$ at a resolution of $4 \mathrm{~cm}^{-1}$. The raw TCD powders were diluted with $\mathrm{KBr}$ mixing powders at $1 \%$ and separately pressed to self-supporting disks $[13,14]$.

2.2.3. LC-MS/MS. The quantitative analyses of the raw TCD and TCD nanoparticles were monitored using a triple quadrupole mass spectrometer (API300, MDS Sciex, USA) equipped with an APCI interface [15]. The samples were dissolved in methanol. The mass spectrometer was operated in electrospray ionization (ESI) negative ion mode with the ion spray voltage $5500 \mathrm{~V}$. The other parameters were as follows: collision gas was at $6 \mathrm{~L} / \mathrm{min}$, the curtain gas was at $10 \mathrm{~L} / \mathrm{min}$, and the nebulizing gas was at $12 \mathrm{~L} / \mathrm{min} . \mathrm{m} / z$ ranged from 100 amu to $1000 \mathrm{amu}$.

2.2.4. XRD. The crystal forms of the samples were detected using an X-ray diffractometer (Philips, Xpert-Pro; Netherlands). The current and voltage using $\mathrm{Cu} \mathrm{K} \alpha \mathrm{l}$ radiation were $30 \mathrm{~mA}$ and $40 \mathrm{kV}$, respectively. The angular range was scanned from $5^{\circ}$ to $50^{\circ}$ of $2 \theta$, with a step size of $0.02^{\circ}$ at a rate of $5^{\circ} / \mathrm{min}[16]$.

2.2.5. DSC. Thermal analysis was carried out using DSC (TA instruments, model DSC 204) for raw TCD and TCD nanoparticle. Analysis was performed for $5.0 \mathrm{mg}$ samples at temperature from 35 to $200^{\circ} \mathrm{C}$ at a heating rate of $10^{\circ} \mathrm{C} / \mathrm{min}$ under $\mathrm{N}_{2}$ atmosphere.

2.2.6. GC. The residual amounts of ethanol in the TCD nanoparticle were analyzed using an Agilent 7890A gas chromatograph (Agilent Technologies, Palo Alto, CA, USA) with an HP-5 5\% phenyl methyl siloxane capillary column $(30.0 \mathrm{~m} \times 320 \mathrm{~mm} \times 0.25 \mu \mathrm{m}$, nominal $)$ equipped with a G1540N-210 flame ionization detector. TCD nanoparticle $(0.2 \mathrm{~g})$ was weighed and placed into a $10 \mathrm{~mL}$ volumetric flask, and $10 \mathrm{~mL}$ of ethanol was added. The flask was shaken by ultrasonic agitation at $20^{\circ} \mathrm{C}$. The sample was then centrifuged at $10000 \mathrm{rpm}$ for $10 \mathrm{~min}$, and $1.0 \mu \mathrm{L}$ of supernatants was injected into the GC injection port. The GC analysis conditions of ethanol were as follows. The oven temperature was initially maintained at $40^{\circ} \mathrm{C}$ for $12 \mathrm{~min}$, raised at a rate of $10^{\circ} \mathrm{C} / \mathrm{min}$ to $240^{\circ} \mathrm{C}$, and held for $10 \mathrm{~min}$. The injector temperature was $200^{\circ} \mathrm{C}$, and the detector temperature was $280^{\circ} \mathrm{C}$. Nitrogen gas was used as carrier gas at a flow rate of $25 \mathrm{~mL} / \mathrm{min}$, and $5 \mu \mathrm{L}$ samples were manually injected in split mode with a split ratio of $20: 1$. Hydrogen gas and air flow rates were 30 and $400 \mathrm{~mL} / \mathrm{min}$, respectively.

2.2.7. HPLC Condition. HPLC was analyzed with a Waters chromatograph (Waters Co., USA). The chromatographic column was Diamonsil $\mathrm{C}_{18}$ reverse-phase column $(250 \mathrm{~mm}$ $\times 4.6 \mathrm{~mm}, 5 \mu \mathrm{m}$; China). The mobile phase consisted of $30: 70(\mathrm{v} / \mathrm{v})$ mixtures of acetonitrile and $0.1 \%$ phosphoric acid solution. The column temperature was set at room temperature. The injection volume and flow rate were $20 \mu \mathrm{L}$ and $1.0 \mathrm{~mL} / \mathrm{min}$, respectively. The effluent was monitored at $278 \mathrm{~nm}$.

2.2.8. Surfactant Residual Analysis. The method for determination of the amount of HPMC in TCD nanoparticles was established in this part as follows: $3 \mathrm{~mL}$ of $0.5 \mathrm{mg} / \mathrm{mL}$ TCD nanoparticles water solution and $5 \mathrm{~mL}$ diphenylamine solution (1.4\% diphenylamine, 39.7\% glacial acetic acid, and $58.9 \%$ concentrated hydrochloric acid) (w/w) were added to the comparison tubes. After mixing, the comparison tubes were heated in a boiling water bath for $30 \mathrm{~min}$ and finally cooled to room temperature in ice bath [17]. The absorbance spectrum was scanned using a 5500PC ultraviolet spectrophotometer (Yuanxi Instruments, China), at wavelengths from 500 to $700 \mathrm{~nm}$. The HPMC comparisons were tested and content was calculated using a standard curve based on absorbance at $634 \mathrm{~nm}$; then the content of HPMC which remained could be determined.

2.2.9. Dissolution Rate Test. The dissolution rate of the TCD powders was analyzed. The paddle speed and solution temperature were set at $75 \mathrm{rpm}$ and $37.0 \pm 0.5^{\circ} \mathrm{C}$, respectively. Artificial gastric juice ( $\mathrm{pH} 1.5$ ) with $0.5 \%$ (w/w) Tween-80 was used as dissolution medium. The raw TCD $(5 \mathrm{mg})$ and physical mixture of raw TCD with HPMC and mannitol (containing $5 \mathrm{mg}$ TCD) and TCD nanoparticles (containing $5 \mathrm{mg}$ TCD) were added to the dialysis bag with $200 \mathrm{~mL}$ artificial gastric juice as dissolution medium. Samples $(5 \mathrm{~mL})$ were withdrawn each time at 5, 10, 20, 30, 45, 60, 90, and $120 \mathrm{~min}$. The same volume dissolution medium was then supplemented. The filtrate was directly injected into an HPLC system and assayed for TCD concentration.

2.2.10. DPPH Free Radical Scavenging. The DPPH radical scavenging assay is commonly employed to evaluate the antioxidant abilities to scavenge free radicals [18]. Using the $\mathrm{DPPH}$ free radical method is advantageous in evaluating antioxidant effectiveness because it is more stable than the hydroxyl and superoxide radicals [19]. The antioxidant of the raw TCD and TCD nanoparticles was determined by $\mathrm{DPPH}$ radical scavenging assay by UV spectrophotometry. The raw and TCD nanoparticle were dissolved at a certain concentration with deionized water, mixed by ultrasound 
shaking for $1 \mathrm{~h}$, and centrifuged. Different concentrations of $1.5 \mathrm{~mL}$ sample solution were added to $1.5 \mathrm{~mL} 0.1 \mathrm{~mol} / \mathrm{L} \mathrm{DPPH}$ in $95 \%$ ethanol. The mixture was mixed, vibrated, and placed at room temperature for $30 \mathrm{~min}$. Then the absorbance of the samples was tested at a wavelength of $517 \mathrm{~nm}$. The clearance rate was calculated as follows: clearance rate $(\%)=\left(\left(A_{0}-\right.\right.$ A) $\left./ A_{0}\right) * 100$, where $A_{0}$ is the absorbance of blank and $A$ is the absorbance of sample; $1.5 \mathrm{~mL}$ of distilled water added to $1.5 \mathrm{~mL}$ of $95 \%$ ethanol to zero is blank [20].

\subsubsection{In Vitro Transdermal Penetration. A transdermal dif-} fusion device (Henan Instrument Co., Ltd., China) was used to analyze the in vitro transdermal penetration by using the improved Franz diffusion cell method [21]. The receiving pool volume was $5 \mathrm{~mL}$, and the effective area was $0.95 \mathrm{~cm}^{2}$. The Sprague-Dawley (SD) rat (Animal Ethics Committee of Harbin Medical University) weighed approximately 200 g. A blade was used to shave off abdominal hair after the rat was sacrificed. The abdominal skin was cut, and the subcutaneous fat layer and the connective tissue were peeled off and cleaned with saline water. The abdominal skin of SD rat was punched into two same round pills. The cut layer of the rat skin was placed facing the two supply rooms and fixed in the middle of the diffusion pool. The TCD nanosuspension and raw drug suspension $(5 \mathrm{~mL})$ were added to the two supply rooms with a concentration of $1 \mathrm{mg} / \mathrm{mL}$. PBS $(5 \mathrm{~mL})$ was later added to both receiving rooms. The dermis and receiving liquid were allowed to contact completely. The diffusion pool was maintained at $37 \pm 0.5^{\circ} \mathrm{C}$ and stirred at $100 \mathrm{rpm}$. Samples $(1 \mathrm{~mL})$ were tested at $0.5,1,1.5,2,3,4,5$, and $6 \mathrm{~h}$ after the 30 min balance time; meanwhile, the same amount of PBS was added. The samples were processed by centrifugation, and the supernatant was obtained and detected by HPLC at least three times.

The in vitro cumulative penetration amount of per unit area was calculated by the following formula:

$$
Q_{n}=\frac{\left(C_{n} V_{n}+\sum C_{i} V_{i}\right)}{A},
$$

where $C_{n}$ is the drug concentration of $n(n=1,2,3,4,5$, 6,7 , and 8) sampling point, $C_{i}$ is the drug concentration of $i(i=n-1)$ sampling point, $V_{n}$ is the volume of receiving pool size $(5 \mathrm{~mL})$, and $V_{i}$ is the sample volume $(1 \mathrm{~mL}) . A$ is the quantity of reagent [22].

\subsection{Bioavailability Study}

2.3.1. Animals and Sample Preparation. Twelve male Sprague-Dawley (SD) rats weighing $200 \mathrm{~g}$ to $250 \mathrm{~g}$ were used in the study. The rats were randomly divided into two groups of six. The rats were housed under standard conditions of temperature, humidity, and light with food and water provided ad libitum. They were allowed to acclimatize in the laboratory for at least 1 week prior to the experiment. Before administration, the animals were fasted overnight with free access to water and were given ether anesthesia before the test. The raw TCD $(15 \mathrm{mg}$ ) and TCD nanoparticles (containing $15 \mathrm{mg}$ TCD), which contain 30\% mannitol and 2.25\% HPMC, were dispersed in $3 \mathrm{~mL}$ of deionized water. Two groups of male rats $(n=6)$ were administered with an oral dose (20 mg/kg by gavage). Heparin $(50 \mathrm{mg}$ ) was dissolved in $5 \mathrm{~mL}$ of deionized water, shaken, and mixed by ultrasound shaking. Blood samples obtained by puncture of the orbital venous sinus were collected into heparinized tubes before and at $0.08,0.25,0.5,1,1.5,2,2.5,3,5,8,12$, and $24 \mathrm{~h}$ after oral administration. The samples were immediately centrifuged at $3000 \mathrm{rpm}$ for $10 \mathrm{~min}$, and aliquots of plasma were stored at $-40^{\circ} \mathrm{C}$ until additional extraction and analysis.

2.3.2. Preparation of Plasma Samples. Frozen samples were thawed at room temperature and treated as follows. Each $300 \mu \mathrm{L}$ of aliquot of plasma sample was diluted by $900 \mu \mathrm{L}$ of ethanol, shaken for $2 \mathrm{~min}$, and centrifuged at $10000 \mathrm{rpm}$ for $10 \mathrm{~min}$. The supernatant solution was directly injected into the HPLC system. The analysis conditions were the same as described above. The concentration of the drug was calculated according to the peak area and standard cure. The oral bioavailability of the TCD nanoparticle was calculated by the area under the plasma concentration-time curve (AUC).

\section{Results and Discussion}

3.1. Impact of Various Factors on the MPS of TCD Nanosuspension and Optimization Study. The optimization experiments were carried out separately at 1, 3, 5, 10, 20, and $30 \mathrm{~min}$. Other conditions, such as the volume ratio of antisolvent to solvent, the amount of surfactant, the concentration of TCD-ethanol solution, and the stirring intensity, were maintained at $4: 1,0.3 \%, 150 \mathrm{mg} / \mathrm{mL}$, and $1000 \mathrm{rpm}$, respectively. Figure 2(a) shows that no notable change occurred on the MPS of the TCD nanoparticle. The supersaturation of the TCD nanoparticle within $0 \mathrm{~min}$ to $10 \mathrm{~min}$ became larger because an antisolvent was added to the solvent during liquid antisolvent precipitate process. Therefore, the MPS within $0 \mathrm{~min}$ to $10 \mathrm{~min}$ was relatively large. By contrast, within $10 \mathrm{~min}$ to $30 \mathrm{~min}$, the nanoparticle was stabilized. The supersaturation of the TCD solution increased to the maximum value with longer stirring time and then remained unchanged. This induced that the MPS was reduced and finally maintained. Therefore, the optimal precipitation time of the TCD nanoparticle was determined at $10 \mathrm{~min}$.

The concentration of TCD-ethanol solution has a significant impact on the MPS. The optimization experiments were carried out at TCD solution concentrations of 30,50, 100,150 , and $170 \mathrm{mg} / \mathrm{mL}$. The stirring time, the volume ratio of antisolvent to solvent, the amount of surfactant, and the stirring intensity were separately fixed at $10 \mathrm{~min}, 4: 1$, $0.3 \%$, and $1000 \mathrm{rpm}$, respectively. The result in Figure 2(b) shows that the particle exhibited agglomeration and the large particle size of $261.37 \mathrm{~nm}$ was caused by the rapid particle precipitation when the concentration of TCD solution was $30 \mathrm{mg} / \mathrm{mL}$. However, the MPS of $130 \pm 12.5 \mathrm{~nm}$ was obtained when the concentration of TCD solution was $170 \mathrm{mg} / \mathrm{mL}$. In general, high supersaturation degree indicates small particle size of crystals $[23,24]$. Therefore, the optimal concentration of TCD-ethanol solution was determined at $170 \mathrm{mg} / \mathrm{mL}$. 


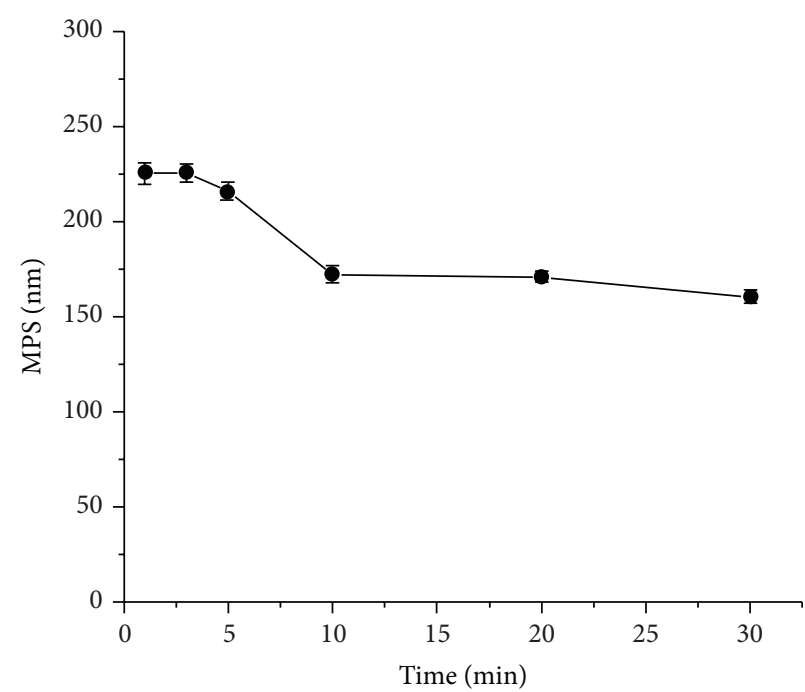

(a)

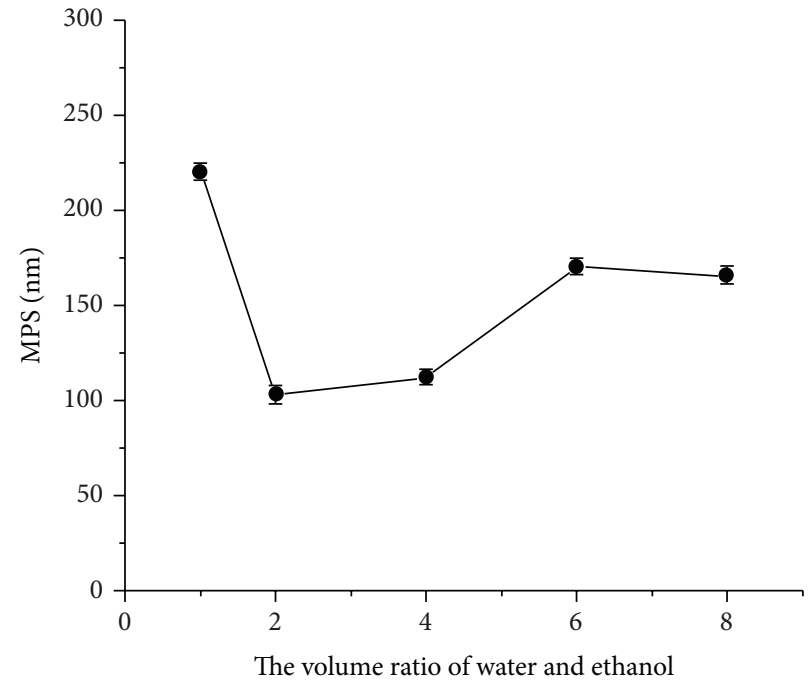

(c)

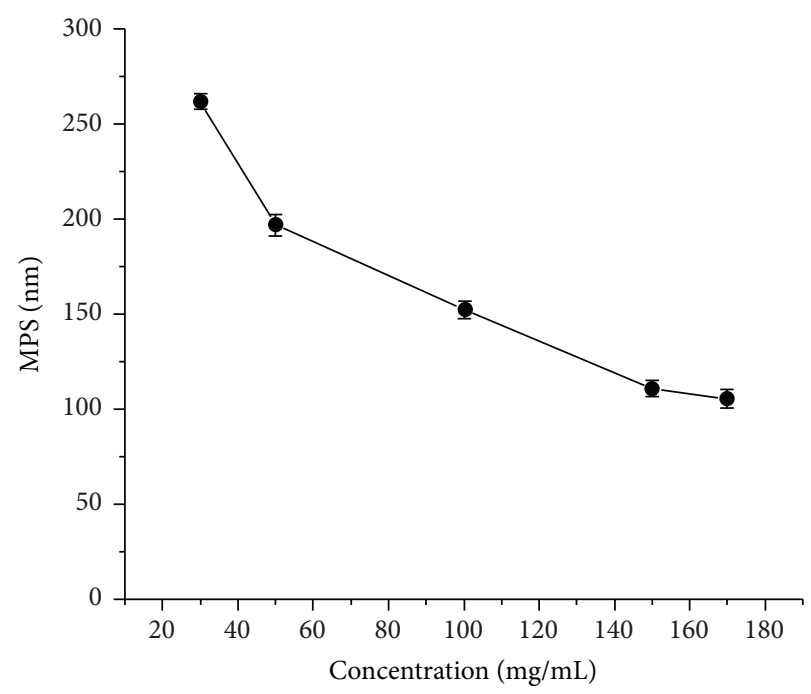

(b)

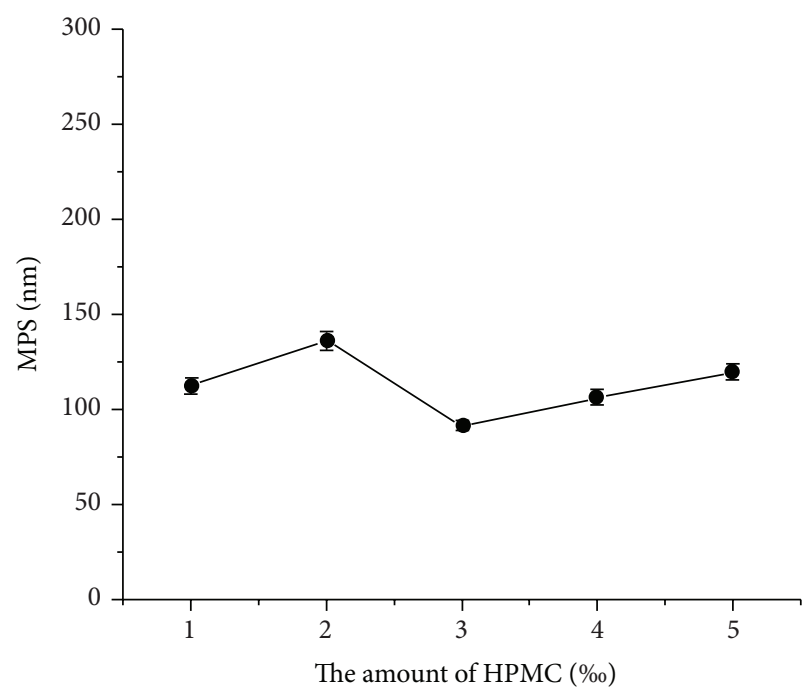

(d)

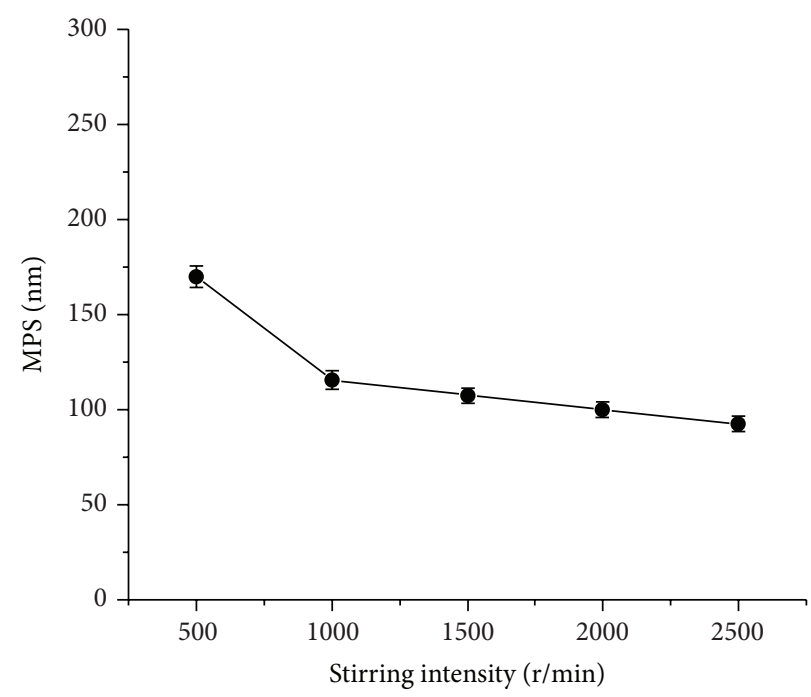

(e)

Figure 2: The effect of each parameter on the MPS of TCD nanoparticle. (a) Precipitation time. (b) Concentration of TCD solution. (c) The volume ratio of antisolvent to solvent. (d) The amount of surfactant. (e) Stirring intensity $(n=3)$. 
Figure 2(c) shows the effect of volume ratio of antisolvent to solvent on the MPS of the TCD nanoparticle. The optimization experiments were carried out at a volume ratio of antisolvent to solvent of $1,2,4,6$, and 8 . The MPS of TCD solution was achieved at minimum, and particle size distribution was uniformed when the volume ratio of antisolvent to solvent was 2; however, high yield was not achieved at this ratio. The volume ratio of antisolvent to solvent of 4 was the optimal condition when yield is considered.

HPMC, a nonionic surfactant, was selected as the surfactant during liquid antisolvent precipitate. This surfactant can stabilize the TCD nanoparticle and prevent the occurrence of reunion phenomenon. The optimization experiments were carried out at the surfactant of TCD solution of $0.1 \%, 0.2 \%$, $0.3 \%, 0.4 \%$, and $0.5 \%$. Figure $2(\mathrm{~d})$ shows the effects of the amount of surfactant on the MPS of the drug. The MPS of the drug achieved minimum when the amount of surfactant was $0.3 \%$. The amount of surfactant was not the key factor on the MPS of drugs when it was controlled from $0.1 \%$ to $0.5 \%$. However, the TCD nanoparticle with uniform distribution and regular shape was obtained after adding this surfactant.

Stirring intensity has a significant influence on the MPS of the TCD nanoparticle. The optimization experiments were carried out at the stirring intensity of TCD solution of 500, 1000, 1500, 2000, and $2500 \mathrm{rpm}$. Figure 2(e) clearly shows the effects of stirring intensity on the MPS of the TCD nanoparticle. When the stirring intensity was $2500 \mathrm{rpm}$, the minimum MPS of the TCD nanoparticle was obtained. The MPS of the product decreased with increasing stirring intensity. High speed stirring strengthened micromixing of liquid phases and more uniform mixing of the solution. This phenomenon promotes the rapid generation of nuclei and uniform growth of particles. Thus, better particle size distribution of the TCD nanoparticle can be obtained. The optimal stirring intensity was determined at $2500 \mathrm{rpm}$.

Based on the single-factor experiment, the optimum conditions of liquid antisolvent precipitate were as follows: stirring time was $10 \mathrm{~min}$, concentration of TCD-ethanol solution was $170 \mathrm{mg} / \mathrm{mL}$, volume ratio of antisolvent to solvent was 4 , amount of HPMC was $0.3 \%(\mathrm{v} / \mathrm{v})$, and stirring intensity was $2500 \mathrm{rpm}$. Under the above conditions, the TCD nanoparticle with a MPS of $130 \pm 12.5 \mathrm{~nm}$ was obtained. All the subsequent characteristics of the optimum samples were obtained under this condition.

3.2. Morphology and MPS of TCD Nanoparticle. Figure 3(a) shows a SEM image of raw TCD. The raw TCD particles are cuboid structure crystals with length varying from $5 \mu \mathrm{m}$ to $50 \mu \mathrm{m}$. The SEM image of the TCD nanoparticle powder has a nearly spherical shape by liquid antisolvent precipitate under the optimum conditions from Figure 3(b). As seen from Figure 3(c), the TCD nanoparticle has a uniform distribution with a MPS of $130 \pm 12.5 \mathrm{~nm}$. In summary, we can obtain the TCD nanoparticle of small particle size with uniform distribution by liquid antisolvent precipitate.

3.3. Chemical Structure of TCD Nanoparticle. The molecular structures of the raw TCD and TCD nanoparticle were examined through FT-IR spectroscopy from $500 \mathrm{~cm}^{-1}$ to $4000 \mathrm{~cm}^{-1}$. The FT-IR spectra of the raw TCD and TCD nanoparticle without mannitol exhibited no changes in the TCD molecular structure before and after the precipitation (Figure 4(a and $\mathrm{c})$ ). The band assignments were as follows: $3418.50 \mathrm{~cm}^{-1}$ (associate $\mathrm{O}-\mathrm{H}$ stretching vibration) and $1687.14 \mathrm{~cm}^{-1}(\mathrm{C}=\mathrm{C}$ stretching vibration). Only the TCD nanoparticle with mannitol showed the different spectra peak at $3265.485 \mathrm{~cm}^{-1}$, which is because of mannitol characteristic absorption. The chemical structures of the two TCD samples were further assessed by using negative ion mode LC-MS to determine the molecular weights. As shown in Figure 5, no change occurred in molecular weight. Calculated from the mass spectrum, the molecular weights of the raw TCD and TCD nanoparticles were 148.1 and 148.2, respectively, which are the same as the molecular weight of 148.16 reported in literature. Combining the results of FT-IR and LC-MS, we determined that the chemical structure of TCD did not change before and after antisolvent.

3.4. Crystal Structure of TCD Nanoparticle. To determine whether the drug crystal structure changed after liquid antisolvent precipitate processing, we performed XRD and DSC analyses [25]. Figure 6 shows the XRD results of three TCD powders. Several distinct peaks of raw TCD at diffraction angles of $2 \theta=9.618^{\circ}, 18.710^{\circ}, 19.574^{\circ}, 22.730^{\circ}$, $25.284^{\circ}, 27.314^{\circ}, 29.418^{\circ}, 31.860^{\circ}$, and $33.776^{\circ}$ revealed that the raw drug was present in crystalline form. However, both TCD nanoparticles and TCD nanoparticles with mannitol showed only several weak peaks at $2 \theta=5.560^{\circ}, 18.372^{\circ}$, and $16.982^{\circ}$ compared with raw TCD. These peaks disappeared or decreased, demonstrating that the crystal structure of the three samples was different. Figure 7 shows the DSC results of three TCD powders. The endothermic melting peaks of raw TCD and TCD nanoparticle were at $134.7^{\circ} \mathrm{C}$ and $133.6^{\circ} \mathrm{C}$, respectively, which decreased $1.1^{\circ} \mathrm{C}$ compared to the raw TCD. The TCD nanoparticle has a small endothermic peak at $85^{\circ} \mathrm{C}$, which could be the HPMC endothermic peak. And the TCD nanoparticle with mannitol has a melting peak at $160^{\circ} \mathrm{C}$ because of mannitol. The results indicated that the TCD nanoparticle had more amorphous state after liquid antisolvent precipitate processing. The amorphous state of the TCD nanoparticle exhibited a higher dissolution rate and bioavailability than the crystalline state of the TCD nanoparticle. The former state played a short role and increased the antioxidant capacity in vitro.

3.5. Solvent Residue Analysis. Solvent residue problems must also be considered in pharmaceutical products. In this study, the TCD nanoparticle was prepared by the liquid antisolvent precipitate process using the International Conference on Harmonization (ICH) class III solvent ethanol with low toxicity as the solvent. The peak time of ethanol solution was at $2.945 \mathrm{~min}$. A regression equation between peak $(y)$ and ethanol concentration $(x)$ can be fitted as $y=371.94 x-$ $91.803\left(R^{2}=0.9997\right)$. According to the regression equation, the residual ethanol content in the TCD nanoparticle is $112 \mathrm{ppm}$. Given that the ICH limit for ethanol in class III 


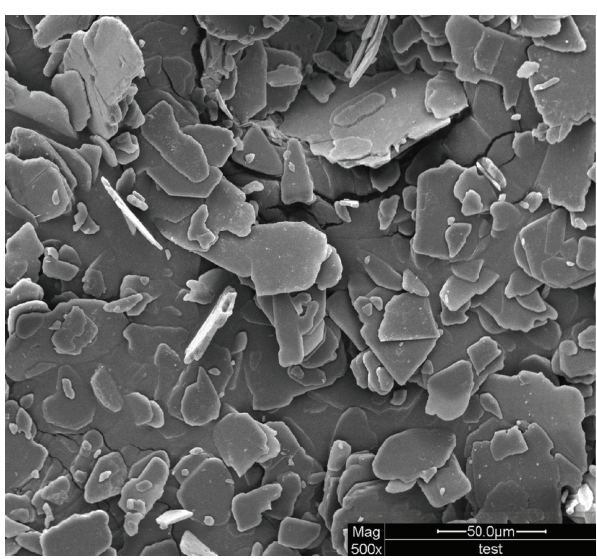

(a)

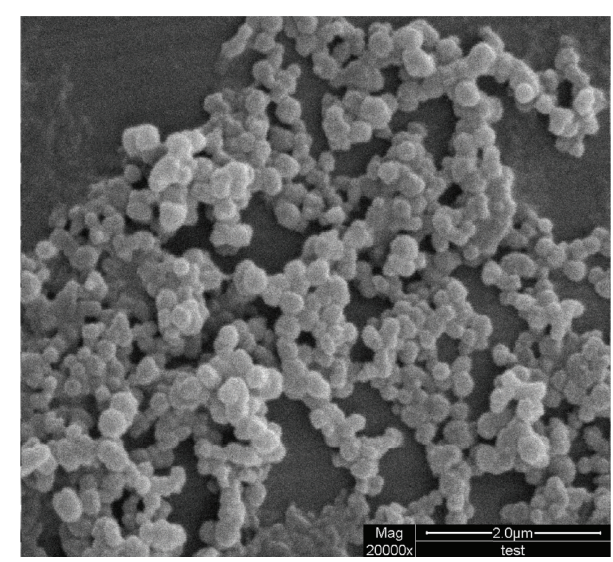

(b)

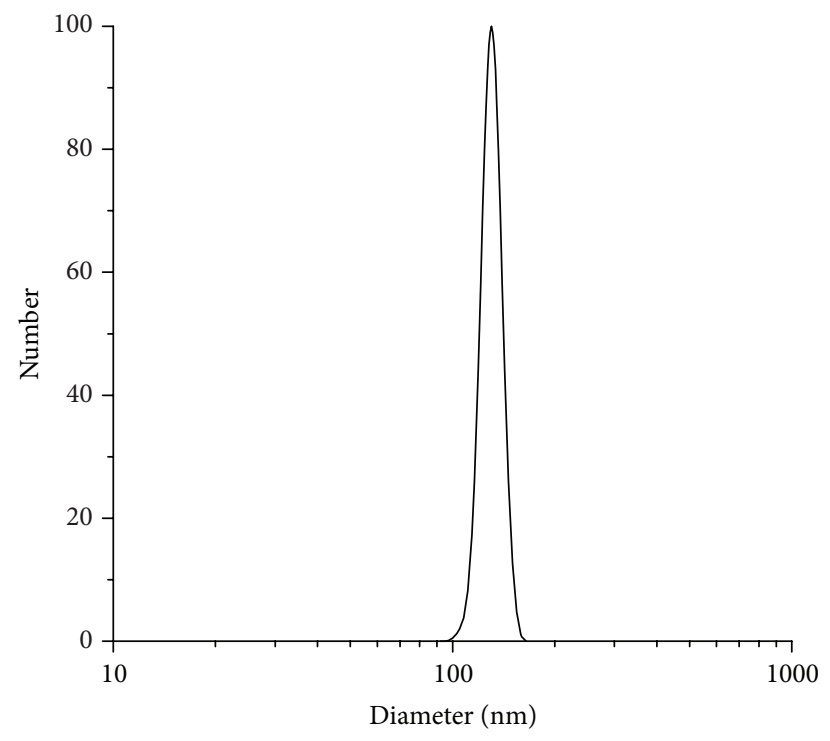

(c)

Figure 3: Morphology and size distribution of TCD. (a) SEM images of raw TCD powders. (b) SEM images of TCD nanoparticle. (c) The size distribution of TCD nanoparticle suspension.

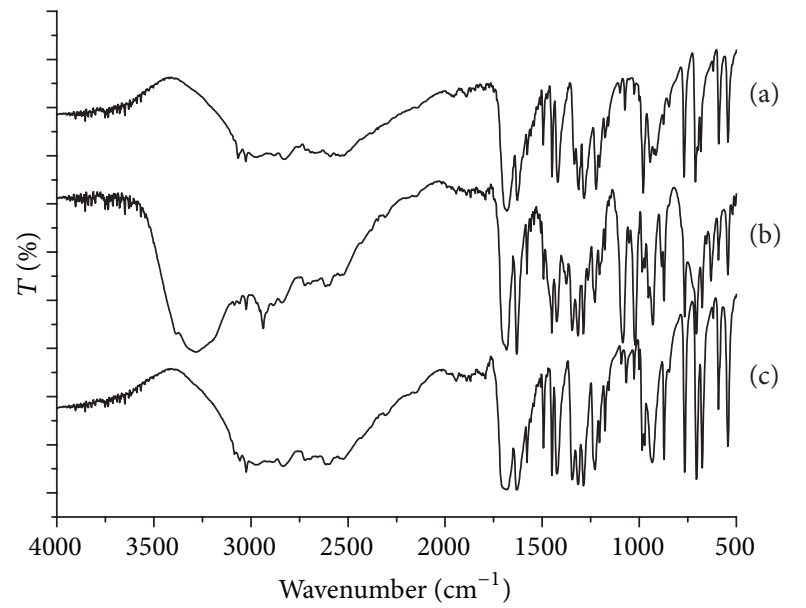

FIGURE 4: FT-IR spectra of three TCD powders. (a) Raw TCD. (b) TCD nanoparticle. (c) TCD nanoparticle without mannitol. solvents is $5000 \mathrm{ppm}$ or $0.5 \%$, the TCD nanoparticle met the $\mathrm{ICH}$ requirement and is suitable for pharmaceutical use.

3.6. Surfactant Residual Analysis. The absorbance spectrum of TCD samples at wavelengths from 500 to $700 \mathrm{~nm}$ is shown in Figure 8. From the spectrum of HPMC standard solution, a regression equation between absorbance $(y)$ at $634 \mathrm{~nm}$ and concentration $(x)$ can be fitted as $y=0.004 x+0.0323\left(R^{2}=\right.$ $0.9989)$. According to the regression equation, the residual HPMC content in TCD nanoparticle was $2.25 \%$.

3.7. Dissolution Rate. Figure 9 presents the dissolution profile of the raw TCD, TCD nanoparticles, and physical mixture of raw TCD with HPMC and mannitol. The dissolution rate of TCD nanoparticle was faster than those of raw TCD and physical mixture. After $2 \mathrm{~h}$, the dissolution rates of TCD nanoparticle, physical mixture, and raw TCD were $86.0 \pm$ 


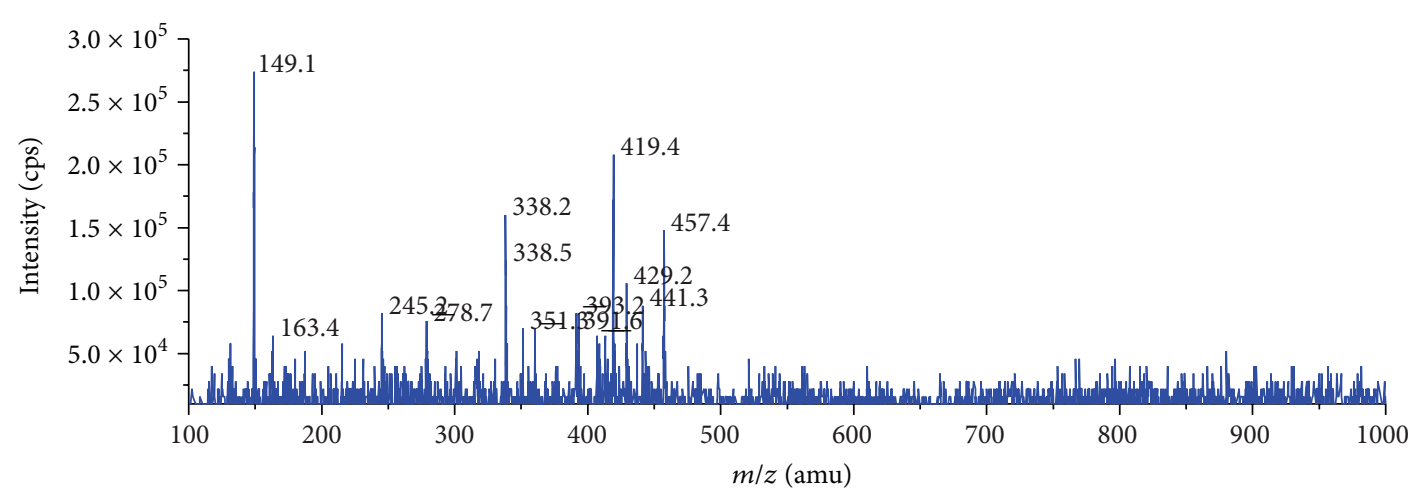

(a)

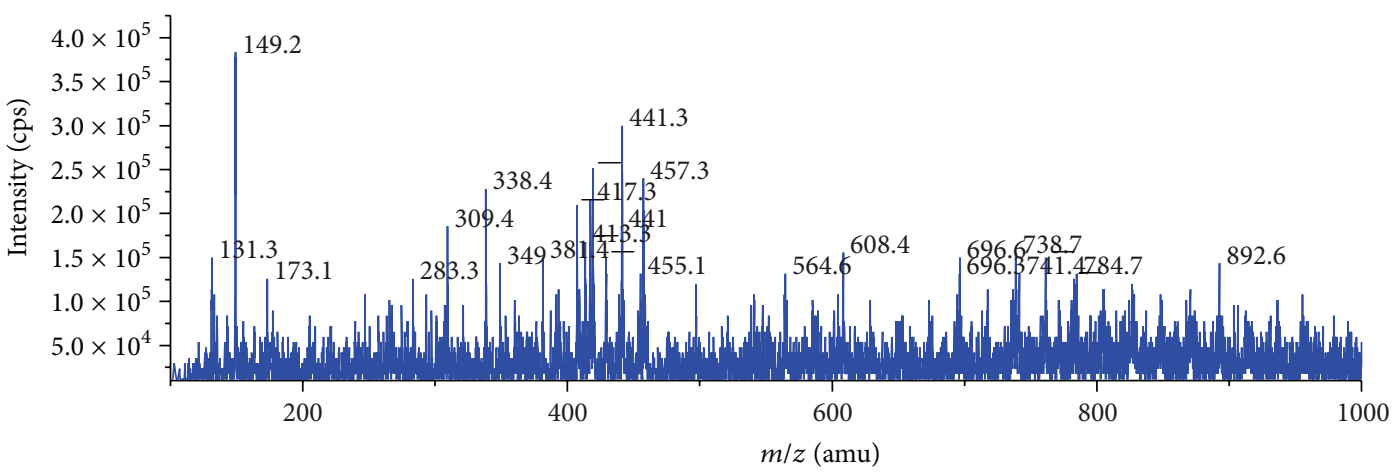

(b)

FIgURE 5: The LC-MS spectra of (a) raw TCD and (b) TCD nanoparticle.

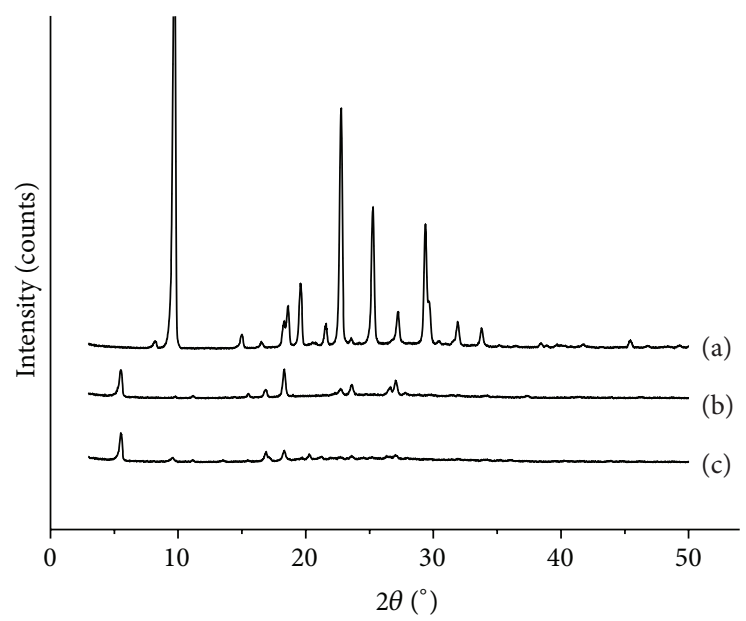

FIGURE 6: XRD patterns of three TCD powders. (a) Raw TCD. (b) TCD nanoparticle. (c) TCD nanoparticle without mannitol.

$3.8 \%, 73.1 \% \pm 0.6 \%$, and $51.5 \pm 1.3 \%$, respectively. It means that the auxiliary material was not the key factor in improving the dissolution rate of TCD. According to the Noyes-Whitney equation, dissolution rate can be proportionally increased by reducing the particle size to increase the interfacial surface area [26]. In addition, the solubility of the TCD nanoparticle powder can also be increased by reducing the particle size based on the Oswald-Freundlich equation [27]. Therefore, the partially amorphous and smaller drug particles exhibited higher dissolution rate and better bioavailability than crystals.

3.8. Antioxidant Activity. The results of in vitro antioxidant capability are shown in Figure 10 by DPPH free radical scavenging activity assay method. The clearance rate of TCD nanoparticle is higher than that of raw TCD nanoparticle 


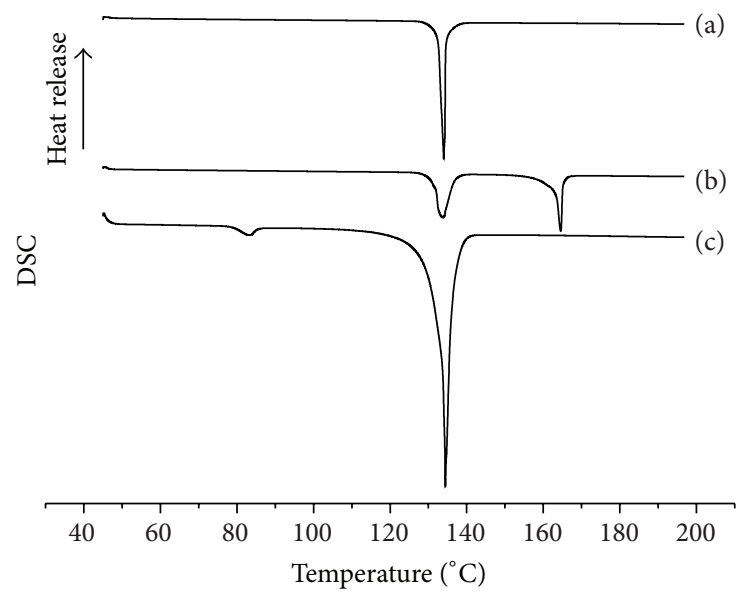

FIGURE 7: DSC curves of three TCD powders. (a) Raw TCD. (b) TCD nanoparticle. (c) TCD nanoparticle without mannitol.

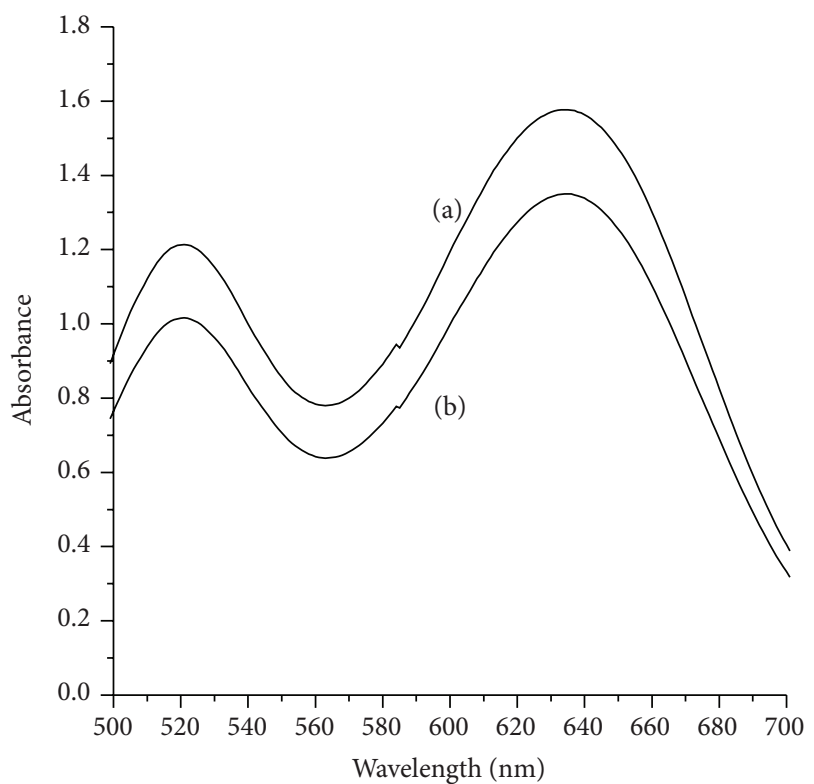

Figure 8: The absorbance of TCD samples at wavelengths from 500 to $700 \mathrm{~nm}$. (a) HPMC standards. (b) TCD nanoparticle.

at the same concentration. $\mathrm{IC}_{50}$ values revealed the drug concentration needed when $50 \%$ free radicals were cleared. The strong ability of the drugs to scavenge free radicals indicates small $\mathrm{IC}_{50}$ values. The $\mathrm{IC}_{50}$ values of the raw TCD and TCD nanoparticles were 19.241 and $13.912 \mathrm{mg} / \mathrm{mL}$, respectively. Thus TCD nanoparticle has a higher DPPH radical scavenging activity than raw TCD.

3.9. In Vitro Transdermal Penetration. In vitro transdermal penetration is an important indicator of drug, for external use. The result of transdermal penetration in vitro is shown in Figure 11. The cumulative penetration amounts of the TCD nanoparticle and raw TCD were $105.5 \pm 4.94 \mu \mathrm{g} /\left(\mathrm{cm}^{-2} \cdot \mathrm{h}\right)$ and $58.5 \pm 2.29 \mu \mathrm{g} /\left(\mathrm{cm}^{-2} \cdot \mathrm{h}\right)$, respectively. The former is 1.81 times

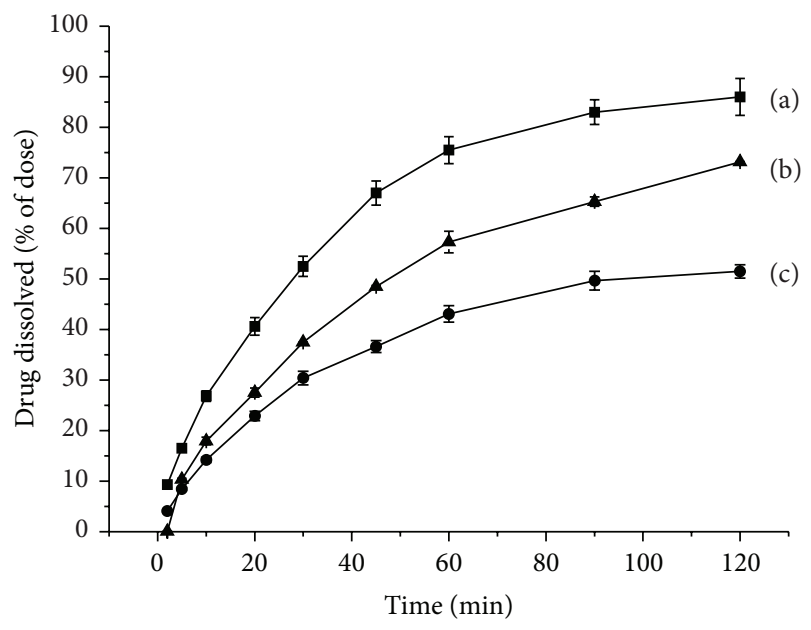

FIGURE 9: Dissolution profiles of three TCD powders $(n=3)$. (a) TCD nanoparticle. (b) Physical mixture of raw TCD with HPMC and mannitol. (c) Raw TCD.

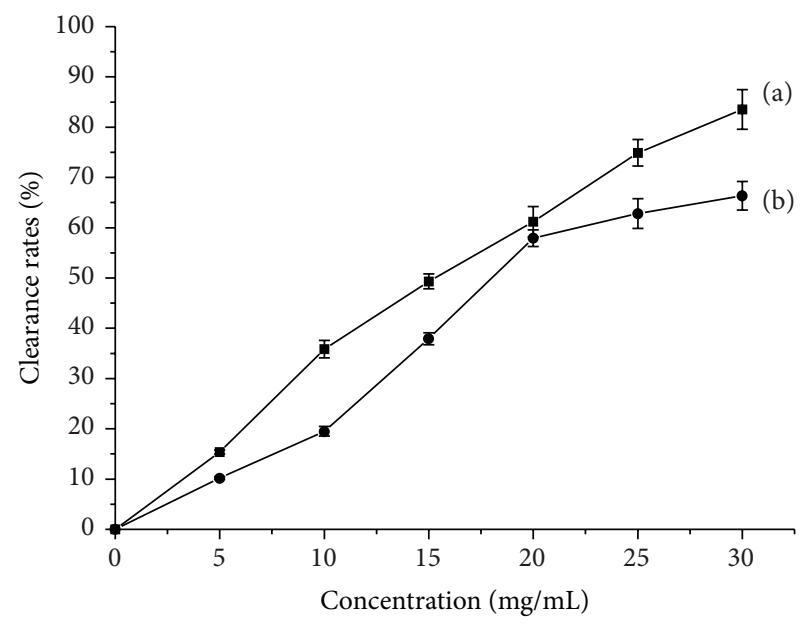

FIGURE 10: Clearance rates diagrams of TCD nanoparticle and raw TCD $(n=3)$. (a) TCD nanoparticle. (b) Raw TCD.

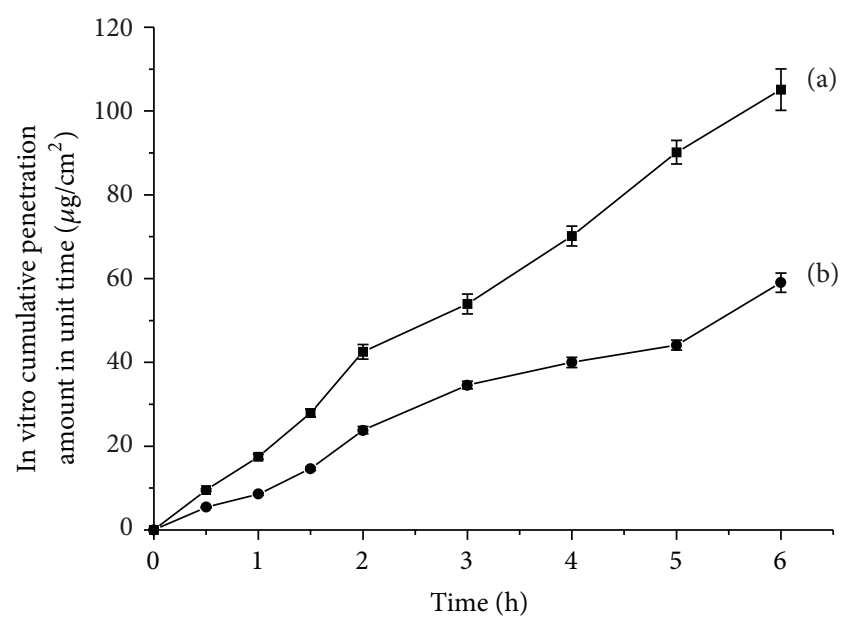

FIGURE 11: In vitro cumulative penetration amount of TCD nanoparticle and raw TCD $(n=3)$. (a) TCD nanoparticle. (b) Raw TCD. 


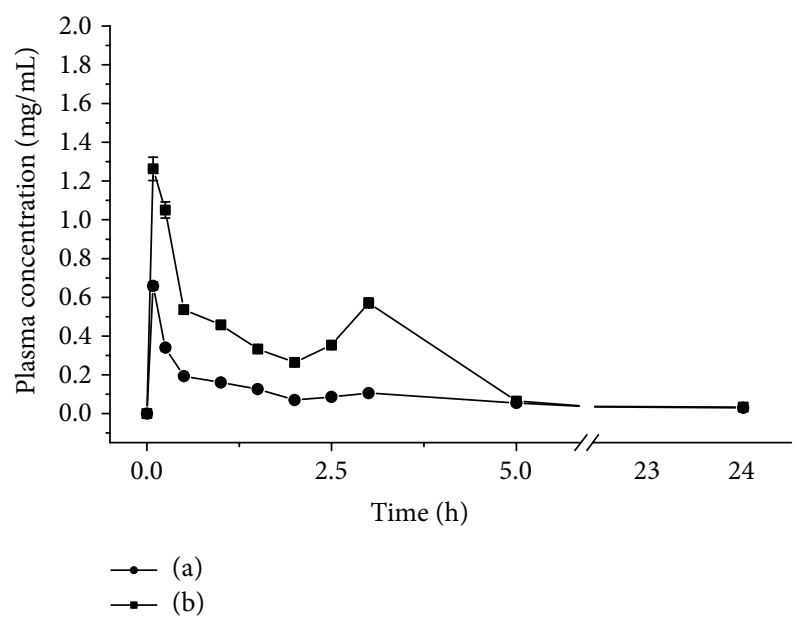

FIGURE 12: The plasma concentration-time curve of TCD nanoparticle and raw TCD $(n=3)$. (a) Raw TCD. (b) TCD nanoparticle.

of the latter. This fact indicates that increase of penetration rate may be due to the decrease of the MPS of TCD.

3.10. Bioavailability Analysis. Figure 12 shows the bioavailability results of the raw and TCD nanoparticles. The TCD concentration in rat plasma of the TCD nanoparticle group was always higher than that of the raw TCD group. These of TCD nanoparticles and raw TCD groups reached the maximum of 1.26 and $0.65 \mathrm{mg} / \mathrm{mL}$ at 5 min after taking the drugs, respectively. The bioavailability parameters are listed in Table 2. The total bioavailability within 24 hours of the TCD nanoparticles was 2.16-fold higher than that of raw TCD. The elimination rate constant $(K)$ of raw TCD and TCD nanoparticles is $0.991 / \mathrm{h}$ and $1.031 / \mathrm{h}$, respectively. The elimination half-life $\left(t_{1 / 2 K}\right)$ of raw TCD and TCD nanoparticles is $0.70 \mathrm{~h}$ and $0.67 \mathrm{~h}$, respectively. That can expound the onset time of TCD nanoparticles which is faster than that of raw TCD. The reasons for these clear differences of bioavailability parameters between the two drugs may be due to higher specific surface area, amorphous state, and smaller particle size.

\section{Conclusions}

In this study, liquid antisolvent precipitate process was successfully employed to prepare a TCD nanoparticle for the enhancement of dissolution rate, antioxidant activity, transdermal penetration, and bioavailability. Using ethanol as solvent and deionized water with HPMC as antisolvent was the best combination. The results indicate that the minimum MPS of the TCD nanoparticle was obtained when the concentration of TCD-ethanol solution, the volume ratio of antisolvent to solvent, the precipitation time, the amount of surfactant, and the stirring intensity were $170 \mathrm{mg} / \mathrm{mL}, 4: 1$, $10 \mathrm{~min}, 0.3 \%$, and $2500 \mathrm{rpm}$, respectively. Under the optimum conditions, the MPS of the TCD nanoparticle was achieved at $130 \pm 12.5 \mathrm{~nm}$ by single-factor analysis. The results of SEM indicate that the TCD nanoparticle obtained was small and
TABLE 2: The pharmacokinetic parameters of TCD by oral administration $(n=3)$.

\begin{tabular}{lcc}
\hline & Raw TCD & TCD nanoparticles \\
\hline$K^{\mathrm{a}}(1 / \mathrm{h})$ & $0.99 \pm 0.007$ & $1.03 \pm 0.013$ \\
$K a^{\mathrm{b}}(1 / \mathrm{h})$ & $0.01 \pm 0.00$ & $0.01 \pm 0.00$ \\
$t_{1 / 2 K^{c}}{ }^{\mathrm{d}}(\mathrm{h})$ & $0.70 \pm 0.004$ & $0.67 \pm 0.009$ \\
$T_{\max }{ }^{\mathrm{d}}(\mathrm{h})$ & $0.08 \pm 0.002$ & $0.08 \pm 0.002$ \\
$C_{\max }{ }^{\mathrm{e}}(\mathrm{mg} / \mathrm{L})$ & $0.65 \pm 0.03$ & $1.26 \pm 0.06$ \\
$\mathrm{AUC}_{0-t}{ }^{\mathrm{f}}(\mathrm{mg} / \mathrm{L} * \mathrm{~h})$ & $1.32 \pm 0.04$ & $2.85 \pm 0.08$ \\
$\mathrm{AUMC}_{0-t}{ }^{\mathrm{g}}(\mathrm{mg} / \mathrm{L} * \mathrm{~h})$ & $10.13 \pm 0.47$ & $13.62 \pm 0.67$ \\
$\mathrm{MRT}_{0-t}{ }^{\mathrm{h}}(\mathrm{h})$ & $7.62 \pm 0.22$ & $4.78 \pm 0.15$ \\
\hline
\end{tabular}

${ }^{\mathrm{a}}$ The elimination rate constant.

${ }^{\mathrm{b}}$ The absorption rate constant.

${ }^{\mathrm{c}}$ The elimination half-life.

${ }^{\mathrm{d}}$ Time of peak concentration.

${ }^{\mathrm{e}}$ Peak of maximum concentration.

${ }^{\mathrm{f}}$ Area under the concentration-time profile curve until last observation.

${ }^{\mathrm{g}}$ Area under moment curve computed to the last observation.

${ }^{\mathrm{h}}$ Mean residence time.

spherical. FT-IR, XRD, DSC, and LC-MS data showed that the TCD nanoparticle had the same chemical structure as the raw drug but with lower crystallinity. The TCD nanoparticle exhibited 1.67 times faster dissolution rate and 3.33 times larger solubility than the raw drug. Furthermore, the amounts of residual solvent of the TCD nanoparticle are suitable for pharmaceutical use. The in vitro antioxidant ability of the TCD nanoparticle was 1.81 times higher than that of the raw TCD. The total oral bioavailability within $24 \mathrm{~h}$ of the TCD nanoparticles was 2.16-fold higher than that of raw TCD. These results suggested that TCD nanoparticle might have potential value to become a new oral or transdermal TCD formulation with high bioavailability.

\section{Competing Interests}

The authors declare that they have no competing interests.

\section{Acknowledgments}

The authors would like to acknowledge the financial support from the National Key Technology R\&D Program (2012BAD21B05) and the National Natural Science Foundation of China (no. 21473023). The authors would also like to acknowledge the Heilongjiang Postdoctoral Grant (LBHZ14016).

\section{References}

[1] Y. Ito, B. Borecka, J. Trotter, and J. R. Scheffer, "Control of solidstate photodimerization of trans-cinnamic acid by double salt formation with diamines," Tetrahedron Letters, vol. 36, no. 34, pp. 6083-6086, 1995.

[2] A. Simić, M. D. Soković, M. Ristić, S. Grujić-Jovanović, J. Vukojević, and P. D. Marin, "The chemical composition of some Lauraceae essential oils and their antifungal activities," Phytotherapy Research, vol. 18, no. 9, pp. 713-717, 2004. 
[3] C. Kopp, S. P. Singh, P. Regenhard, U. Müller, H. Sauerwein, and M. Mielenz, "Trans-cinnamic acid increases adiponectin and the phosphorylation of AMP-activated protein kinase through g-protein-coupled receptor signaling in 3T3-L1 adipocytes," International Journal of Molecular Sciences, vol. 15, no. 2, pp. 2906-2915, 2014.

[4] M. Alkhalaf, "Resveratrol-induced growth inhibition in MDAMB-231 breast cancer cells is associated with mitogen-activated protein kinase signaling and protein translation," European Journal of Cancer Prevention, vol. 16, no. 4, pp. 334-341, 2007.

[5] G.-C. Yen, Y.-L. Chen, F.-M. Sun, Y.-L. Chiang, S.-H. Lu, and C.-J. Weng, "A comparative study on the effectiveness of cis- and trans-form of cinnamic acid treatments for inhibiting invasive activity of human lung adenocarcinoma cells," European Journal of Pharmaceutical Sciences, vol. 44, no. 3, pp. 281-287, 2011.

[6] C.-M. Tsai, F.-M. Sun, Y.-L. Chen, C.-L. Hsu, G.-C. Yen, and C.-J. Weng, "Molecular mechanism depressing PMA-induced invasive behaviors in human lung adenocarcinoma cells by cisand trans-cinnamic acid," European Journal of Pharmaceutical Sciences, vol. 48, no. 3, pp. 494-501, 2013.

[7] K. Patra, S. Bose, S. Sarkar et al., "Amelioration of cyclophosphamide induced myelosuppression and oxidative stress by cinnamic acid," Chemico-Biological Interactions, vol. 195, no. 3 , pp. 231-239, 2012.

[8] H. F. Sun, B. Hohl, Y. Z. Cao et al., "Jet mill grinding of portland cement, limestone, and fly ash: impact on particle size, hydration rate, and strength," Cement and Concrete Composites, vol. 44, pp. 41-49, 2013.

[9] L. Miller, "Pharmaceutical purifications using preparative supercritical fluid chromatography," Chimica Oggi-Chemistry Today, vol. 32, no. 2, pp. 23-26, 2014.

[10] Y. G. Zu, N. Li, X. H. Zhao et al., "In vitro dissolution enhancement of micronized L-nimodipine by antisolvent recrystallization from its crystal form $\mathrm{H}$," International Journal of Pharmaceutics, vol. 464, no. 1-2, pp. 1-9, 2014.

[11] D. Murnane, C. Marriott, and G. P. Martin, "Comparison of salmeterol xinafoate microparticle production by conventional and novel antisolvent crystallization," European Journal of Pharmaceutics and Biopharmaceutics, vol. 69, no. 1, pp. 94-105, 2008.

[12] S.-D. Yeo and J.-C. Lee, "Crystallization of sulfamethizole using the supercritical and liquid antisolvent processes," The Journal of Supercritical Fluids, vol. 30, no. 3, pp. 315-323, 2004.

[13] R. Sivaraj, P. K. S. M. Rahman, P. Rajiv, H. A. Salam, and R. Venckatesh, "Biogenic copper oxide nanoparticles synthesis using Tabernaemontana divaricate leaf extract and its antibacterial activity against urinary tract pathogen," Spectrochimica Acta Part A: Molecular and Biomolecular Spectroscopy, vol. 133, pp. 178-181, 2014.

[14] S. Sundar, R. Mariappan, and S. Piraman, "Synthesis and characterization of amine modified magnetite nanoparticles as carriers of curcumin-anticancer drug," Powder Technology, vol. 266, pp. 321-328, 2014.

[15] M. Piller, G. Gilch, G. Scherer, and M. Scherer, "Simple, fast and sensitive LC-MS/MS analysis for the simultaneous quantification of nicotine and 10 of its major metabolites," Journal of Chromatography B, vol. 951-952, no. 1, pp. 7-15, 2014.

[16] Y. Zu, W. Sun, X. Zhao et al., "Preparation and characterization of amorphous amphotericin B nanoparticles for oral administration through liquid antisolvent precipitation," European Journal of Pharmaceutical Sciences, vol. 53, no. 1, pp. 109-117, 2014.
[17] C. Ding, M. Zhang, H. Tian, and G. Li, "Effect of hydroxypropyl methylcellulose on collagen fibril formation in vitro," International Journal of Biological Macromolecules, vol. 52, no. 1, pp. 319-326, 2013.

[18] G. Morales and A. Paredes, "Antioxidant activities of Lampaya medicinalis extracts and their main chemical constituents," BMC Complementary and Alternative Medicine, vol. 14, no. 1, article 259, 2014.

[19] D. Chandrasekar, K. Madhusudhana, S. Ramakrishna, and P. V. Diwan, "Determination of DPPH free radical scavenging activity by reversed-phase HPLC: a sensitive screening method for polyherbal formulations," Journal of Pharmaceutical and Biomedical Analysis, vol. 40, no. 2, pp. 460-464, 2006.

[20] B. Shan, J.-H. Xie, J.-H. Zhu, and Y. Peng, "Ethanol modified supercritical carbon dioxide extraction of flavonoids from Momordica charantia L. and its antioxidant activity," Food and Bioproducts Processing, vol. 90, no. 3, pp. 579-587, 2012.

[21] D. Szura, Ł. Ozimek, M. Przybyło et al., "The impact of liposomes on transdermal permeation of naproxen-in vitro studies," Acta Poloniae Pharmaceutica, vol. 71, no. 1, pp. 145-151, 2013.

[22] A. C. Sintov and I. Greenberg, "Comparative percutaneous permeation study using caffeine-loaded microemulsion showing low reliability of the frozen/thawed skin models," International Journal of Pharmaceutics, vol. 471, no. 1-2, pp. 516-524, 2014.

[23] B. Y. Shekunov and P. York, "Crystallization processes in pharmaceutical technology and drug delivery design," Journal of Crystal Growth, vol. 211, no. 1-4, pp. 122-136, 2000.

[24] A. Y. Lee, I. S. Lee, S. S. Dette, J. Boerner, and A. S. Myerson, "Crystallization on confined engineered surfaces: a method to control crystal size and generate different polymorphs," Journal of the American Chemical Society, vol. 127, no. 43, pp. 1498214983, 2005.

[25] Y. Sert, H. Doğan, A. Navarrete, R. Somanathan, G. Aguirre, and Ç. Çirak, "Experimental FT-IR, Laser-Raman and DFT spectroscopic analysis of 2,3,4,5,6-Pentafluoro-trans-cinnamic acid," Spectrochimica Acta Part A: Molecular and Biomolecular Spectroscopy, vol. 128, pp. 119-126, 2014.

[26] Y. Hattori, Y. Haruna, and M. Otsuka, "Dissolution process analysis using model-free Noyes-Whitney integral equation," Colloids and Surfaces B: Biointerfaces, vol. 102, pp. 227-231, 2013.

[27] R.-L. Tseng and F.-C. Wu, "Analyzing a liquid-solid phase countercurrent two- and three-stage adsorption process with the Freundlich equation," Journal of Hazardous Materials, vol. 162, no. 1, pp. 237-248, 2009. 

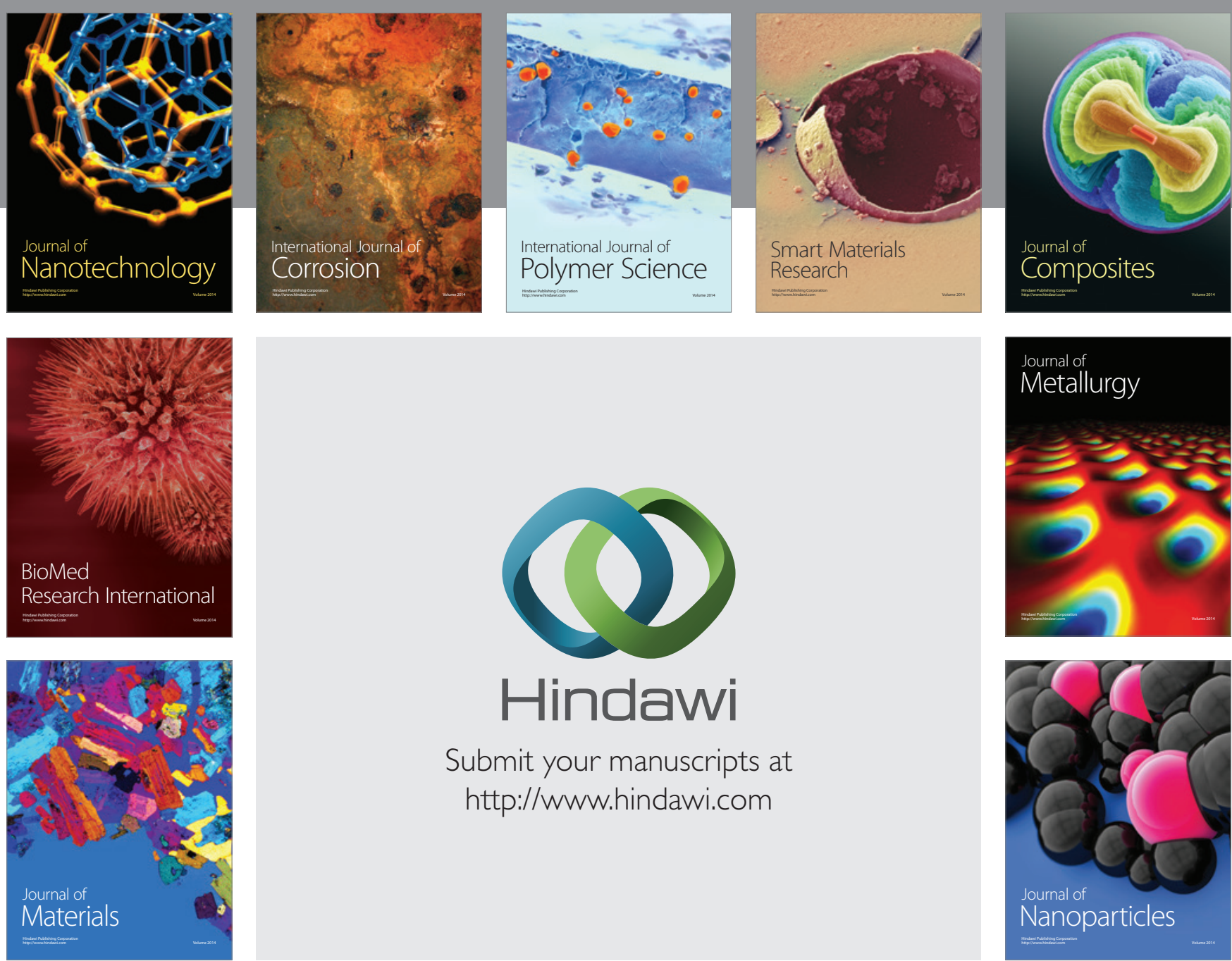

\section{Hindawi}

Submit your manuscripts at

http://www.hindawi.com

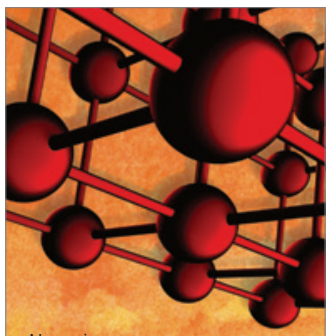

Materials Science and Engineering
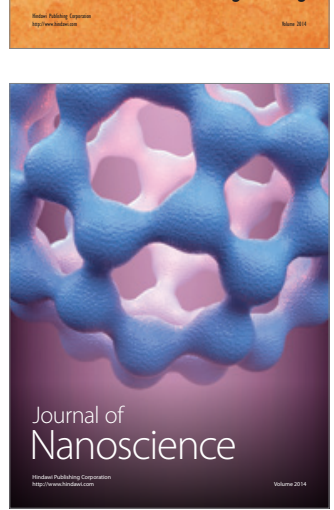
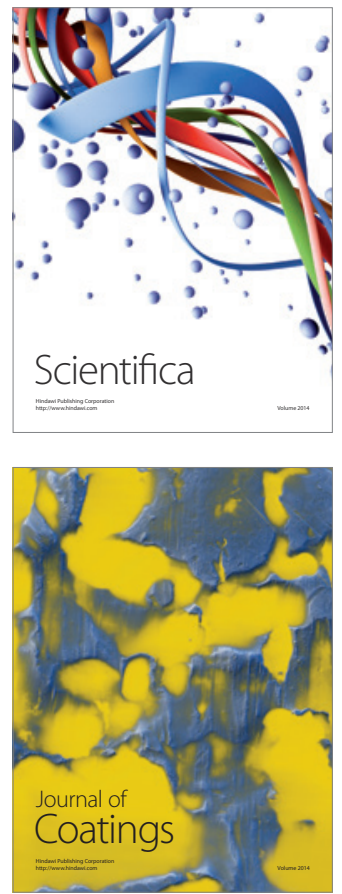
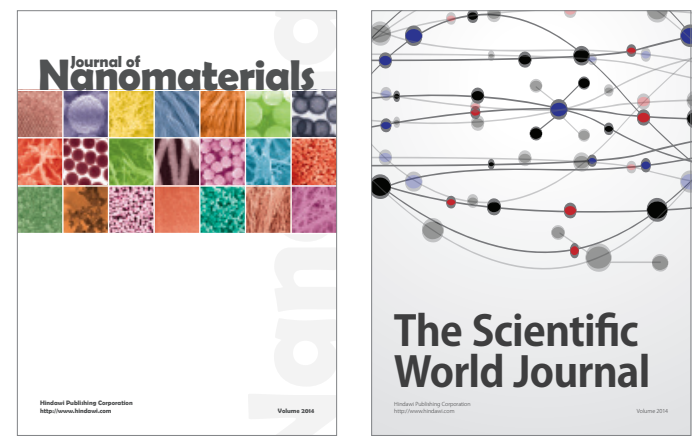

The Scientific World Journal
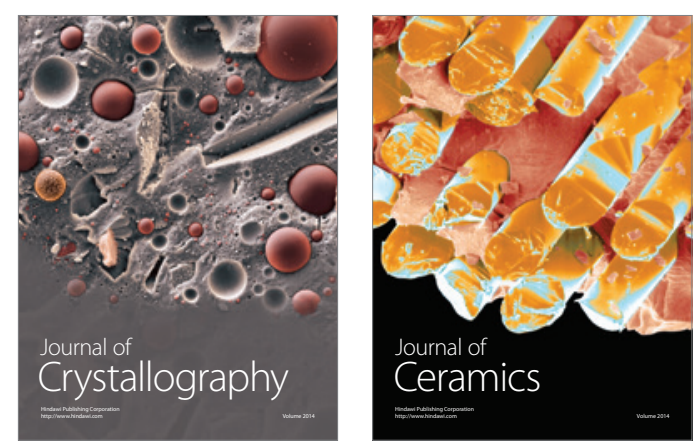
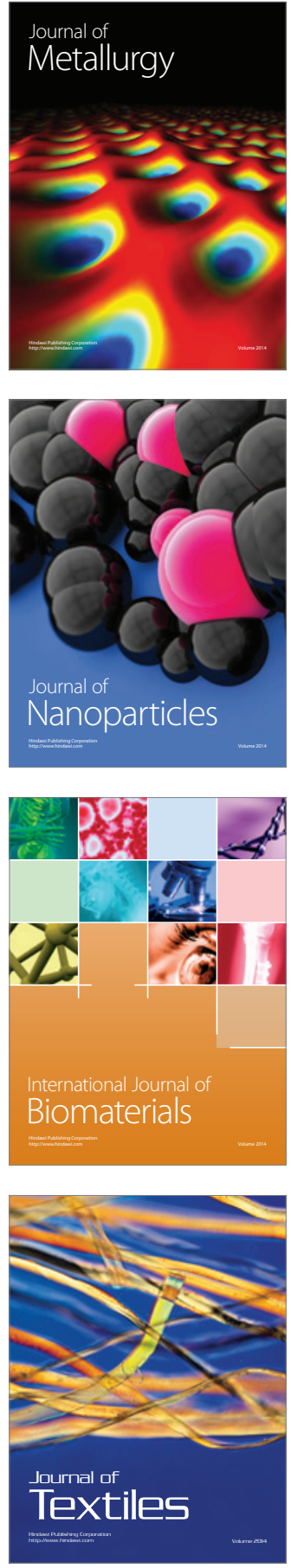